\title{
Similarities across packaging of infant formula, follow-on formula and formula labelled as food for special medical purposes available in mainland GB
}

\author{
R. Conway ${ }^{1}$, S. Esser ${ }^{1}$, A. Smith ${ }^{1}$, A. Steptoe ${ }^{1}$ and C. Llewellyn ${ }^{1}$ \\ ${ }^{1}$ Research Department of Behavioural Science and Health, University College London, London, UK
}

Birth to age 2 is a critical period for establishing lifelong healthy eating habits. The composition, labelling and marketing of formula is governed by Regulation (EU) No 609/2013 and delegated acts, which the UK adopted in 2021 ${ }^{(1)}$. These permit promotion of follow-on formula (FOF, suitable from 6 months) but not infant formula (IF, suitable from birth) or formula labelled as food for special medical purposes (FSMP, suitable for the dietary management of specific conditions e.g. colic). Furthermore, they state that packaging (text, images and colours) of FOF and formula labelled as FSMP should be designed to avoid confusion with IF. Similarity between products promotes brand identity but could result in inappropriate infant feeding. The present study aimed to assess similarities between the packaging of IF, FOF and formula labelled as FSMP.

A scoping review of formula available over the counter in mainland GB between April and October 2020 was undertaken. Images of the front, sides and back of formula products were compiled in a database. Prominent visual aspects of packaging were coded and classified. Five features were examined, based on those previously identified in Department of Health and Social Care (DHSC) guidelines as likely to cause confusion ${ }^{(2)}$. Products received one score point for each feature that was the same (colour of packaging, size and position of logo, image used, position of image, position of text describing product) giving a maximum similarity score of 5 . The higher the similarity score, the more challenging the formula products within a brand range are to distinguish from each other.

In total 71 formula products were identified, and 302 images were coded and examined. This included 18 FOFs, which were compared to 18 same brand IFs. We also analysed 6 formulas labelled as FSMP, that were compared to 6 same brand IFs. A similarity score $\geq 4$ out of 5 was found for $72 \%$ of FOFs and $29 \%$ of formulas labelled as FSMP. In addition, $94 \%$ of IF packs included on-package advertisements for FOF and one quarter (5/18) included nutrition and health claims within advertisements, which are promotional tools and not permitted on IF in order to protect breastfeeding.

FOF and formula labelled as FSMP are sold in packaging which appears similar to IF. These risks infants being given inappropriate formula by mistake. The health and nutrition claims displayed on FOFs may also have a health halo effect on IFs which are not permitted to include such claims. These findings highlight the need for future legislation and guidelines to include clear instructions to ensure packaging for different types of formula is clearly distinguishable and will feed into a qualitative study of formula choices.

\section{Acknowledgements}

This study is funded by the National Institute for Health Research (NIHR) (PR-PRU-0916-21001]. The views expressed are those of the authors and not necessarily those of the NIHR or DHSC.

\section{References}

1. Regulation (EU) No 609/2013 of the European Parliament and of the Council of 12 June 2013. [Available at: https://www.legislation.gov.uk/eur/ 2013/609/contents].

2. Department of Health (2013). DH guidance notes on the infant formula and follow-on formula regulations 2007 (as amended)[Available at: http:// www.dh.gov.uk/publications]. 